\title{
Triggered star formation in the inner wing of the SMC. Two possible supernova explosions in the N83-84-85 region ${ }^{\star}$
}

\author{
E. Bratsolis ${ }^{1,2}$, M. Kontizas ${ }^{2}$, and I. Bellas-Velidis ${ }^{3}$ \\ 1 École Nationale Supérieure des Télécommunications, Département Traitement du Signal et des Images, \\ 46 rue Barrault, 75013 Paris, France \\ e-mail: bratsoli@tsi.enst.fr \\ ${ }^{2}$ Department of Astrophysics, Astronomy and Mechanics, Faculty of Physics, University of Athens, 15783 Athens, Greece \\ 3 National Observatory of Athens, Institute of Astronomy and Astrophysics, Lofos Koufou - P. Penteli, 15236 Athens, Greece
}

Received 1 December 2003 / Accepted 3 May 2004

\begin{abstract}
In this article we study the N83-84-85 region of the inner wing of the SMC. Direct and low-dispersion objective prism plates taken with the $1.2 \mathrm{~m}$ UK Schmidt Telescope have been digitized by the SuperCOSMOS machine. Star counts have been performed for our region in selected luminosity slices in the $U$ filter and isodensity contours have been used to identify the structures with enhanced stellar number density. We find evidence of triggered star formation from massive stars of older to more recent $\mathrm{OB}$ associations. Circular arcs constructed by $\mathrm{O}$ and $\mathrm{B}$ stars have been detected. A study of the population places stars with more recent ages in the groups of the arcs than of their centers. These effects can be explained by supernova explosions. A catalogue of the non-saturated detected OB stars in this region is given.
\end{abstract}

Key words. techniques: image processing - methods: data analysis - stars: formation - galaxies: Magellanic Clouds shock waves

\section{Introduction}

The formation of massive stars is a topic of major interest. Massive stars are often formed in groups, such as OB associations and clusters. Massive stars finish their life by supernova explosions. The interstellar medium (ISM) is continuously stirred by supernova explosions and stellar winds of massive stars. Shock waves from supernova and stellar wind-blown bubbles around $\mathrm{O}$ and $\mathrm{B}$ type star associations generate turbulences in the ISM. Star formation may trigger further star formation in nearby gas if the density is high enough but also star-forming clouds are destroyed by the same effects and shut off star formation. There remains an open question about the density of the ISM and the violence of the effect. A supernova explosion can produce a shell wall or a dense interstellar cloudlet only if the density is high enough.

In this work we use data from digitized plates, using the direct and objective prism, of the SMC focusing on the inner wing. The same region has been studied by Testor \& Lortet (1988). Our study with automated techniques of the objective prism plates (Bratsolis et al. 1998, 2000; Bratsolis 2002) gave OB populations in circular structures. A new study to investigate plates of the same region with slices of different populations and contour detection (Maragoudaki et al. 2001)

$\star$ Table 3 is only available in electronic form at http://www.edpsciences.org showed that the outer populations of OB stars are more recent OBs of the centers. This is evidence of star-formation triggered by supernova explosions. Therefore, there is a strong correlation of our study region with the supernova remnants (SNRs) of ROSAT X-ray observations No. 223 RX J0112.7-7207 and No. 245 RX J0119.4-7301 (Kahabka et al. 1999). These regions were searched for in IRAS and ISO (for lambda 170). The image of the SMC in both cases is of low resolution.

Following the theoretical work of Vishniac (1994), Elmegreen (1994) and Ehlerová et al. (1997) for isolated supernova explosions we can extract a time scale for the supernova explosions and the density of the ISM before the explosion.

\section{The region $\mathbf{N} 83-84-85$ of the SMC}

The region N83-84-85 belongs to the inner wing of the SMC and is of interest because of its OB associations and nebulae. It is evident that there is a correlation between associations like NGC 456, 460a, b and 465 with the nebulae of ionized gas. This region has been studied by different authors in the past (Westerlund 1961; Azzopardi \& Vigneau 1982; Kontizas et al. 1988; Testor \& Lortet 1987; Lortet \& Testor 1988; Dapergolas et al. 1991; Caplan et al. 1996). There are groups of stars with age variations of 4-10 Myr and spatial scales of 30-400 pc. There is also an extended region containing N83-84-85 with a diameter of more than $500 \mathrm{pc}$ and sequential star formation on a scale of $10^{7} \mathrm{yr}$ which seems to be part of a supergiant shell 


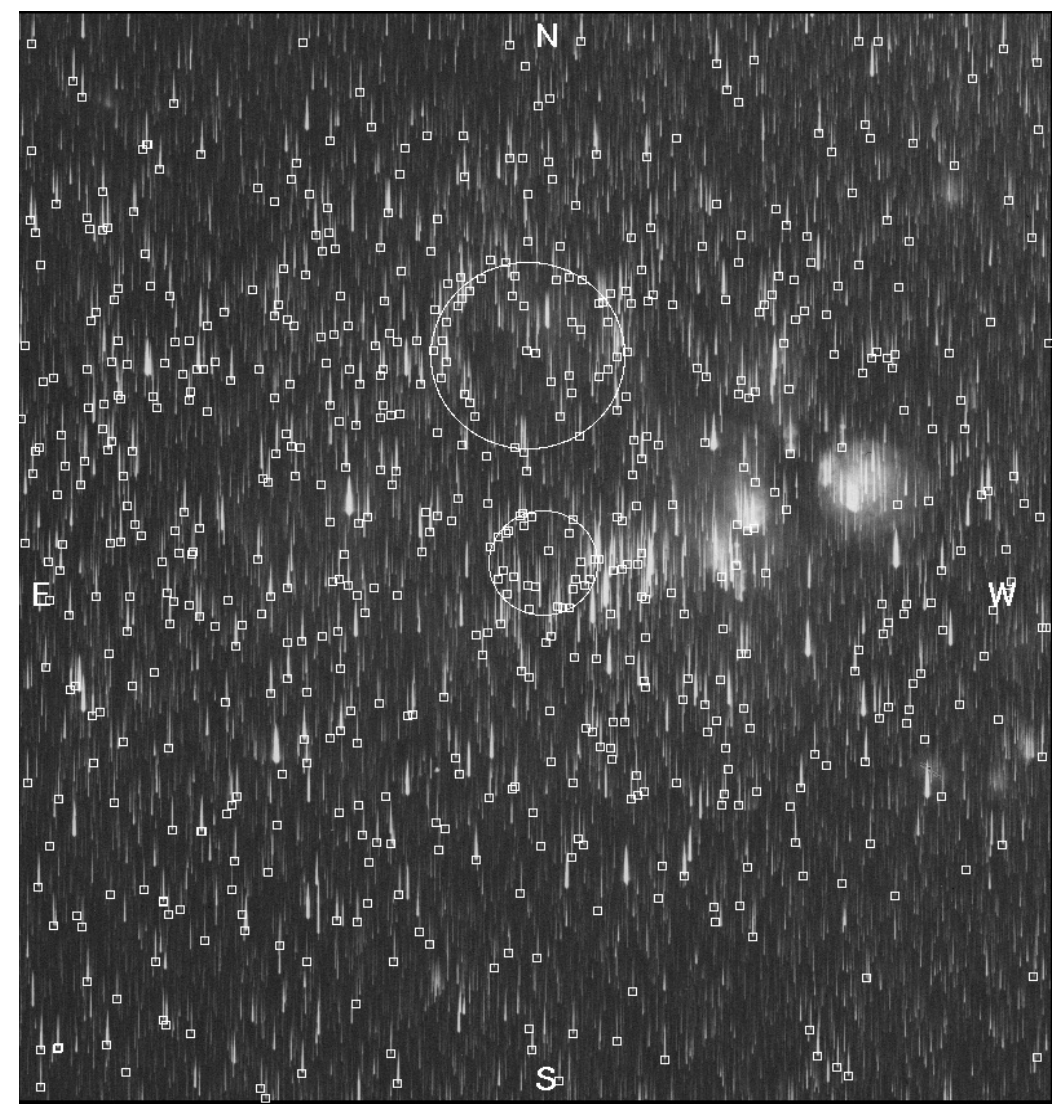

Fig. 1. Positions of OB classified stars with an automated method of correlation. The circles present the regions of SN explosions.

(Westerlund 1961; Staveley-Smith et al. 1997; Stanimirović et al. 1999).

We focus on this region because it seems to show a feedback between OB star formation and the physical properties of the ISM. It suggests that star formation and ISM properties probably are self-regulated. The ISM is continuously stirred by supernova explosions and stellar winds. The shock waves engaged by the supernova accelerate galactic cosmic rays that penetrate deeply into molecular clouds and clumps and heat and ionize them. Far ultraviolet photons are produced by massive star formation and photoinize the less dense surface regions of the molecular cloud and its internal clumps.

\section{Image reduction}

Our test image contains a region of $36.2 \times 39.1 \mathrm{arcmin}^{2}$ of SMC. The scanning pixel size of the SuperCOSMOS measuring machine is $10 \mu \mathrm{m}$ and the plate scale is 67.11 arc$\mathrm{sec} / \mathrm{mm}$. Our image is centered at $\mathrm{RA}_{2000}=1^{\mathrm{h}} 16^{\mathrm{m}}$ and $\operatorname{Dec}_{2000}=-73^{\circ} 20^{\prime}$ and contains a region from $\mathrm{RA}_{2000}=1^{\mathrm{h}} 12^{\mathrm{m}}$ to $\mathrm{RA}_{2000}=1^{\mathrm{h}} 20^{\mathrm{m}}$ and from $\operatorname{Dec}_{2000}=-73^{\circ} 35^{\prime}$ to $\operatorname{Dec}_{2000}=$ $-73^{\circ} 05^{\prime}$. The magnitude limit for classification of our plate is $m_{B}=18.5$.

The detection (DETSP), extraction (EXTSP) and classification (RCORR) was made by automated methods (Bratsolis et al. 1998, 2000; Bratsolis 2002).

The extracted spectra are stored in a two-dimensional file $n \times 128$, where $n$ is the number of detected spectra. Each row of this file is an independent normalized spectrum with a length of 128 pixels. The maximum correlation method has been used to automatically classify the spectra and gave 610 clearly classified OB spectra (Fig. 1).

The same region was also chosen from the direct $U$ plate taken with the UK $1.2 \mathrm{~m}$ Schmidt Telescope. The plate was digitized by the SuperCOSMOS machine and the derived data given for the detected images positions and magnitudes. Isodensity contours have been used to identify the various structures with enhanced stellar density. The contour level separation has been defined by the mean value plus $3 \sigma$ from the local background (Fig. 2). The detected stellar images were divided in various luminosity slices according to their magnitude. The magnitude limit for the $U$ plate is $m_{U}=19.7$, corresponding to the A0 spectral type stars.

The data were separated in four luminosity slices.

The first slice contains main sequence stars with $U<15$ and $-1.5<U-V<-0.8$, corresponding to ages less than $8 \times 10^{6} \mathrm{yr}$ and stars more luminous than B2 spectral type.

The second slice contains main sequence stars with $15<$ $U<16$ and $-1.4<U-V<-0.6$, corresponding to ages of $8 \times 10^{6}-1.2 \times 10^{7} \mathrm{yr}$ and stars of B2 spectral type.

The third slice contains main sequence stars with $16<U<$ 17 and $-1.3<U-V<-0.2$, corresponding to ages of $1.2-3 \times$ $10^{7} \mathrm{yr}$ and stars of late B2 to early B4 spectral type.

The fourth slice contains main sequence stars with $U>17$ and $-1.1<U-V$ corresponding to ages grater than $3 \times 10^{7} \mathrm{yr}$. 

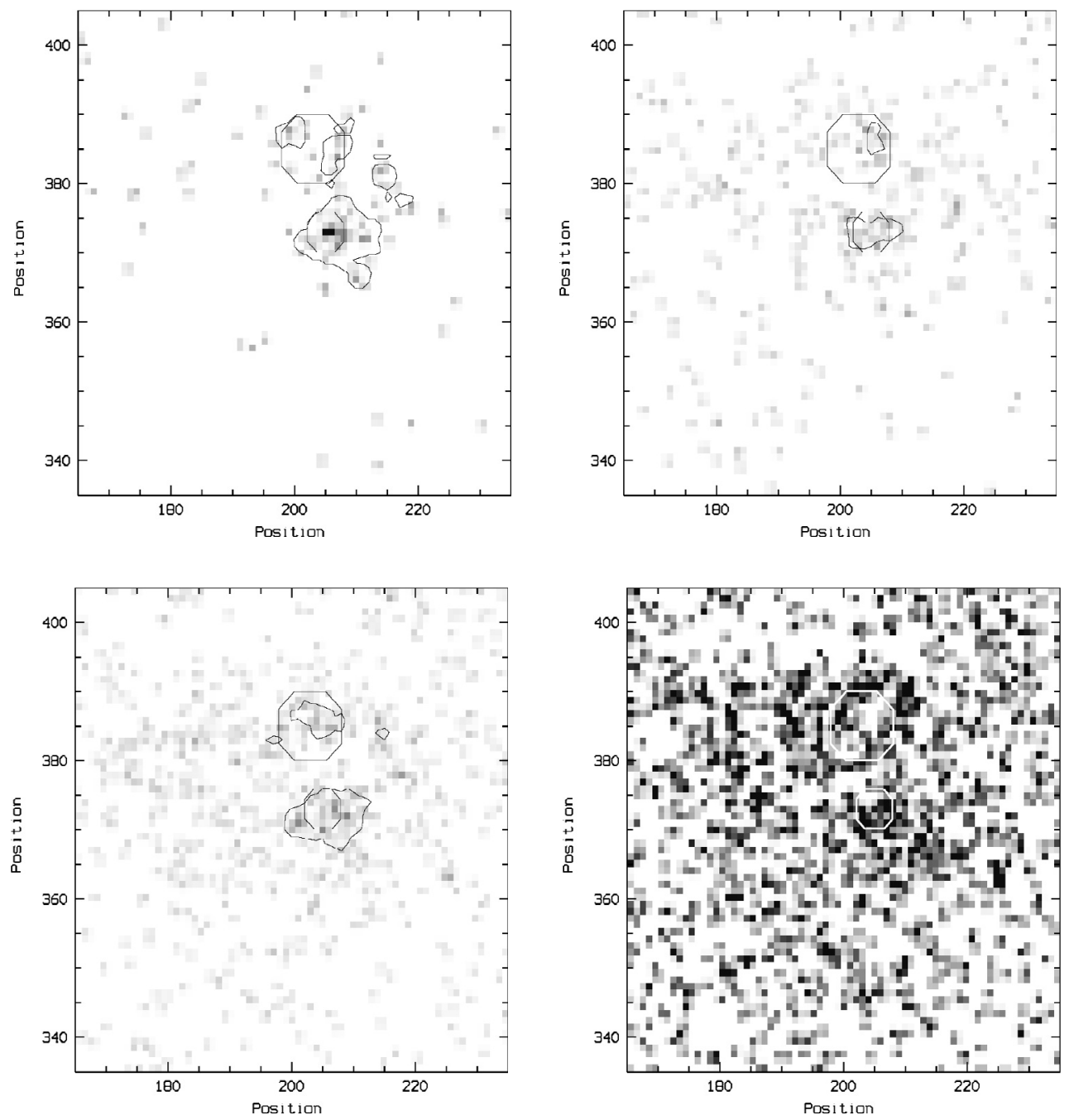

Fig. 2. Upper left: isodensity contours for main sequence OB stars younger than $8 \times 10^{6} \mathrm{yr}$. Upper right: isodensity contours for main sequence B stars with ages between $8 \times 10^{6}$ and $1.2 \times 10^{7}$ yr. Lower left: isodensity contours for main sequence B stars with ages between $1.2 \times 10^{7}$ and $3 \times 10^{7} \mathrm{yr}$. Lower right: density distribution for main sequence B stars with ages older than $3 \times 10^{7} \mathrm{yr}$. The octagons present the regions of SN explosions. Subslices for these star ages do not give any significant contours.

The scale calibration of the $U$ plate, produced for the SMC distance modulus $m-M=19$ and based on theoretical models, is given in Table 1 .

Detected objects in our region (not including the OB stars) are presented by Bica \& Schmitt (1995) in Table 2. The nonsaturated detected OB stars are presented in Table 3.

\section{The evolution of an expanding shell}

Let us accept a simple model of a supernova explosion. The supernova produces a gravitational instability in the ISM and accumulates the gas in an expanding shell. The expanding shell's equations are known as Sedov's equations (Dyson \& Williams 1997), given by

$R(t)=\left(\frac{25}{3 \pi}\right)^{1 / 5}\left(\frac{E_{\mathrm{SN}}}{\rho_{0}}\right)^{1 / 5} t^{2 / 5}$

and

$u_{\mathrm{s}}(t)=\frac{2}{5} \frac{R(t)}{t}$
Table 1. Scale calibration of the $U$ plate for the SMC.

\begin{tabular}{ccc}
\hline \hline Spectral type & $U_{\text {SMC }}$ & Age \\
\hline O5 & 11.9 & $3 \times 10^{6}$ \\
B0 & 13.5 & $4 \times 10^{6}$ \\
B1 & 14.6 & $6 \times 10^{6}$ \\
B2 & 15.4 & $1 \times 10^{7}$ \\
B3 & 16.3 & $1.5 \times 10^{7}$ \\
B4 & 16.7 & $2.8 \times 10^{7}$ \\
B5 & 17.2 & $4 \times 10^{7}$ \\
B6 & 17.5 & $1 \times 10^{8}$ \\
B7 & 17.9 & $1.6 \times 10^{8}$ \\
B8 & 18.4 & $2 \times 10^{8}$ \\
B9 & 18.5 & $3 \times 10^{8}$ \\
A0 & 19.7 & $4 \times 10^{8}$ \\
\hline
\end{tabular}

where $R(t)$ is the radius of the shell and $u_{\mathrm{s}}(t)$ the expansion velocity of the shell at the time $t$ relative to the ambient medium with density $\rho_{0} . E_{\mathrm{SN}}$ is the total supernova explosion energy. 
Table 2. Detected objects in our region according Bica \& Schmitt (1995).

\begin{tabular}{|c|c|c|c|c|c|c|c|c|c|}
\hline Number & $\begin{array}{l}\text { RA(2000) } \\
\text { h m s }\end{array}$ & $\begin{array}{c}\operatorname{Dec}(2000) \\
\circ,{ }^{\prime}\end{array}$ & Name & $x$ Pos. & $y$ Pos. & Type & $\begin{array}{l}\operatorname{Dmax} \\
\left({ }^{\prime}\right)\end{array}$ & $\begin{array}{l}\operatorname{Dmin} \\
\left({ }^{\prime}\right)\end{array}$ & Remarks \\
\hline 887 & 011252 & -730710 & $L 91$ & 223 & 391 & $\bar{C}$ & 1.20 & 1.20 & \\
\hline 894 & 011348 & -731733 & $N G C 456$ & 218 & 376 & $N A$ & 3.80 & 2.90 & \\
\hline 895 & 011350 & -731802 & $S M C-N 83 A$ & 218 & 376 & $N C$ & 0.60 & 0.45 & in $N G C 456$ \\
\hline 896 & 011352 & -731545 & $S M C-N 83 B$ & 217 & 379 & $N C$ & 0.30 & 0.30 & att $N G C 456$ \\
\hline 898 & 011353 & -731556 & $L 61-501$ & 217 & 379 & $N C$ & 0.25 & 0.25 & att $N G C 456$ \\
\hline 899 & 011353 & -732459 & $H W 68$ & 218 & 366 & $C$ & 0.55 & 0.55 & \\
\hline 901 & 011356 & -731553 & MA1779 & 217 & 379 & $N C$ & 0.25 & 0.25 & att $N G C 456$ \\
\hline 902 & 011400 & -731512 & $L 61-502$ & 217 & 380 & $N C$ & 0.30 & 0.30 & \\
\hline 903 & 011402 & -731705 & $S M C-N 83 C$ & 216 & 377 & $N C$ & 0.90 & 0.90 & in $N G C 456$ \\
\hline 906 & 011406 & -731406 & $B 146$ & 216 & 381 & $A$ & 0.75 & 0.75 & att $S M C-N 84 n$ \\
\hline 907 & 011417 & -732242 & $H W 69$ & 215 & 369 & $C$ & 0.55 & 0.55 & \\
\hline 908 & 011418 & -731550 & $S M C-N 84 C$ & 215 & 379 & $N C$ & 0.65 & 0.65 & att $S M C-N 84 n$ \\
\hline 909 & 011422 & -731404 & $S M C-N 84 n$ & 214 & 381 & $N A$ & 4.00 & 3.00 & \\
\hline 910 & 011424 & -731403 & $L 61-505$ & 214 & 381 & $N C$ & 0.35 & 0.30 & in $N 84 n$ \\
\hline 914 & 011437 & $-73 \quad 1624$ & $B S 161$ & 213 & 378 & $N A$ & 1.60 & 1.20 & in $S M C-N 84 s$ \\
\hline 916 & 011438 & -731759 & $N G C 460 n w$ & 213 & 376 & $N A$ & 1.90 & 1.70 & in $S M C-N 84 s$ \\
\hline 917 & 011438 & -731827 & $S M C-N 84 A$ & 213 & 375 & $N C$ & 0.80 & 0.65 & in $N G C 460 n w$ \\
\hline 918 & 011441 & -731830 & $L 61-507$ & 213 & 375 & $N C$ & 0.30 & 0.30 & in $N G C 460 \mathrm{nw}$ \\
\hline 923 & 011445 & -731818 & MA1794 & 212 & 375 & $N A$ & 0.70 & 0.70 & in $N G C 460 \mathrm{nw}$ \\
\hline 924 & 011445 & -732044 & $S M C-N 84 D$ & 212 & 372 & $N C$ & 0.30 & 0.30 & in $N G C 460 \mathrm{se}$ \\
\hline 925 & 011445 & -732254 & $B S 165$ & 212 & 369 & $A C$ & 0.95 & 0.65 & \\
\hline 926 & 011446 & -731854 & $S M C-N 84 s$ & 212 & 374 & $N A$ & 7.50 & 4.00 & \\
\hline 927 & 011447 & -731945 & $S M C-N 84 B$ & 212 & 373 & $N C$ & 0.35 & 0.35 & in NGC460se \\
\hline 928 & 011447 & -732104 & $B S 166$ & 212 & 371 & $N C$ & 0.45 & 0.35 & in NGC460se \\
\hline 929 & 011447 & -732012 & MA1796 & 212 & 372 & $N C$ & 0.20 & 0.20 & in? NGC460se, $P N$ ? \\
\hline 931 & 011452 & -730704 & $B 147$ & 211 & 391 & $A C$ & 1.20 & 1.10 & \\
\hline 932 & 011454 & -732002 & MA1799 & 211 & 373 & $N A$ & 0.60 & 0.55 & in $N G C 460 s e$ \\
\hline 933 & 011454 & -731945 & NGC460se & 211 & 373 & $N A$ & 2.60 & 1.50 & in $S M C-N 84 s$ \\
\hline 934 & 011457 & -731936 & $L 61-512$ & 211 & 373 & $N A$ & 0.50 & 0.35 & in $N G C 460 s e$ \\
\hline 946 & 011532 & -731146 & $S M C-D E M 155$ & 207 & 384 & $N A$ & 1.80 & 1.80 & in $S M C-D E M 157$ \\
\hline 947 & 011542 & -731000 & $H W 72$ & 206 & 386 & $C N$ & 0.55 & 0.45 & in $S M C-D E M 157$ \\
\hline 948 & 011542 & -731946 & $N G C 465$ & 207 & 373 & $A$ & 5.00 & 4.00 & in $S M C-D E M 157$ \\
\hline 949 & 011549 & -732040 & $S k 157$ & 206 & 372 & $C$ & 0.60 & 0.60 & in $N G C 465$ \\
\hline 950 & 011552 & -731856 & $S k 158$ & 206 & 374 & $C$ & 0.70 & 0.60 & in $N G C 465$ \\
\hline 952 & 011609 & -731119 & $S M C-D E M 156$ & 203 & 385 & $N A$ & 2.80 & 2.60 & in $S M C-D E M 157$ \\
\hline 954 & 011621 & -732012 & $S M C-D E M 157$ & 203 & 372 & $N A$ & 22.00 & 17.00 & \\
\hline 956 & 011648 & -730936 & $H W 74$ & 199 & 387 & $C$ & 0.55 & 0.50 & in $S M C-D E M 158$ \\
\hline 957 & 011649 & -730920 & $S M C-D E M 158$ & 199 & 387 & $N A$ & 1.80 & 1.50 & in $S M C-D E M 157$ \\
\hline 958 & 011659 & -731206 & $S M C-D E M 159$ & 198 & 383 & $N A$ & 1.50 & 0.80 & in $S M C-D E M 157$ \\
\hline 959 & 011730 & -733409 & $H W 75$ & 197 & 352 & $C A$ & 0.90 & 0.80 & \\
\hline 961 & 011931 & -730538 & $B 156$ & 183 & 392 & $C$ & 0.65 & 0.45 & \\
\hline
\end{tabular}

After the linear analysis of Elmegreen (1994) and Vishniac (1994) in a uniform medium with gravitation constant $G$, we have that an expanding shell with surface density $\Sigma$ and velocity dispersion in the shell $c$ has an instantaneous maximum growth rate $\omega$ given by

$\omega=-\frac{3 u_{\mathrm{s}}}{R}+\sqrt{\frac{u_{\mathrm{s}}^{2}}{R^{2}}+\left(\frac{\pi G \Sigma}{c}\right)}$.

If $\rho$ and $\rho_{0}$ are the densities of the perturbed and unperturbed medium respectively, then they are connected to the dimensionless quantity $\mathcal{M}$ by the formula (Efremov \& Elmegreen 1998)

$\frac{\rho}{\rho_{0}}=\left(\frac{u_{\mathrm{s}}}{c}\right)^{2}=\mathcal{M}^{2}$

(4) $m(R)=\frac{4 \pi \rho_{0} R^{3}(t)}{3}$
At the time $t_{\mathrm{b}}$, when $\omega>0$ or

$\xi=\frac{\sqrt{8} u_{\mathrm{s}} c}{\pi G R \Sigma}<1$

the perturbation grows.

We now use the same logic as Ehlerová et al. (1997) for isolated supernova explosions and we obtain the radius

$$
\begin{aligned}
R(t)= & 83.2\left(\frac{E_{\mathrm{SN}}}{10^{51} \mathrm{erg}}\right)^{1 / 5}\left[\left(\frac{\mu}{1.3}\right)^{-1}\left(\frac{n_{0}}{\mathrm{~cm}^{-3}}\right)^{-1}\right]^{1 / 5} \\
& \left(\frac{t}{\mathrm{Myr}}\right)^{2 / 5} \mathrm{pc}
\end{aligned}
$$

the total mass 
and the surface density

$\Sigma(t)=\frac{m(R)}{4 \pi R^{2}(t)}$.

From Eqs. (8), (7) and (6) we have

$$
\begin{aligned}
\Sigma(t)= & 0.91\left(\frac{E_{\mathrm{SN}}}{10^{51} \mathrm{erg}}\right)^{1 / 5}\left[\left(\frac{\mu}{1.3}\right)^{4}\left(\frac{n_{0}}{\mathrm{~cm}^{-3}}\right)^{4}\right]^{1 / 5} \\
& \left(\frac{t}{\mathrm{Myr}}\right)^{2 / 5} M_{\odot} / \mathrm{pc}^{2} .
\end{aligned}
$$

From Eqs. (5), (2) and (9) we have

$\xi(t)=0.89 \frac{c}{G} \frac{1}{\left(\rho_{0}^{4} E_{\mathrm{SN}}\right)^{1 / 5}} t^{-7 / 5}$.

The instability time $t_{\mathrm{b}}$ is estimated by the condition

$\xi\left(t_{\mathrm{b}}\right)=1$

and is

$$
\begin{aligned}
t_{\mathrm{b}}= & 21.9\left(\frac{c}{1 \mathrm{~km} \mathrm{~s}^{-1}}\right)^{5 / 7}\left(\frac{n_{0}}{\mathrm{~cm}^{-3}}\right)^{-4 / 7}\left(\frac{\mu}{1.3}\right)^{-4 / 7} \\
& \left(\frac{E_{\mathrm{SN}}}{10^{51} \mathrm{erg}}\right)^{-1 / 7} \mathrm{Myr} .
\end{aligned}
$$

The radius $R$ and the expansion velocity $u_{\mathrm{s}}$ of the shell at $t_{\mathrm{b}}$ are

$$
\begin{aligned}
R\left(t_{\mathrm{b}}\right)= & 286\left(\frac{c}{1 \mathrm{~km} \mathrm{~s}^{-1}}\right)^{2 / 7}\left(\frac{n_{0}}{\mathrm{~cm}^{-3}}\right)^{-3 / 7}\left(\frac{\mu}{1.3}\right)^{-3 / 7} \\
& \left(\frac{E_{\mathrm{SN}}}{10^{51} \mathrm{erg}}\right)^{1 / 7} \mathrm{pc}
\end{aligned}
$$

and

$$
\begin{aligned}
u_{\mathrm{s}}\left(t_{\mathrm{b}}\right)= & \frac{2}{5} \frac{R\left(t_{\mathrm{b}}\right)}{t_{\mathrm{b}}}=5.22\left(\frac{c}{1 \mathrm{~km} \mathrm{~s}^{-1}}\right)^{-3 / 7}\left(\frac{n_{0}}{\mathrm{~cm}^{-3}}\right)^{1 / 7}\left(\frac{\mu}{1.3}\right)^{1 / 7} \\
& \left(\frac{E_{\mathrm{SN}}}{10^{51} \mathrm{erg}}\right)^{2 / 7} \mathrm{~km} \mathrm{~s}^{-1}
\end{aligned}
$$

The estimation of fragmentation time is given by

$\int_{t_{\mathrm{b}}}^{t_{\mathrm{f}}} \omega(t) \mathrm{d} t=1$

From Eqs. (3) and (5) we obtain

$\omega=\frac{u_{\mathrm{s}}}{R}\left(-3+\sqrt{1+\frac{8}{\xi^{2}(t)}}\right)$

and from Eqs. (11) and (10)

$\xi(t)=\left(\frac{t}{t_{\mathrm{b}}}\right)^{-7 / 5}$.

From Eqs. (15), (17), (16) and (2) we have

$$
\int_{t_{\mathrm{b}}}^{t_{\mathrm{f}}} \frac{-3+\sqrt{1+8\left(\frac{t}{t_{\mathrm{b}}}\right)^{14 / 5}}}{t} \mathrm{~d} t=1
$$

and

$$
\int_{1}^{x_{\mathrm{f}}} \frac{-3+\sqrt{1+8 x^{14 / 5}}}{x} \mathrm{~d} x=\frac{5}{2}
$$

where

$x_{\mathrm{f}}=t_{\mathrm{f}} / t_{\mathrm{b}}$.

The numerical solution of (19) gives $x_{\mathrm{f}} \approx 2.47$. From Eqs. (12) and (20) we have

$$
\begin{aligned}
t_{\mathrm{f}}= & 54.1\left(\frac{c}{1 \mathrm{~km} \mathrm{~s}^{-1}}\right)^{5 / 7}\left(\frac{n_{0}}{\mathrm{~cm}^{-3}}\right)^{-4 / 7}\left(\frac{\mu}{1.3}\right)^{-4 / 7} \\
& \left(\frac{E_{\mathrm{SN}}}{10^{51} \mathrm{erg}}\right)^{-1 / 7} \mathrm{Myr} .
\end{aligned}
$$

From Eqs. (21), (6) and (2) we obtain

$$
\begin{aligned}
R\left(t_{\mathrm{f}}\right)= & 410.6\left(\frac{c}{1 \mathrm{~km} \mathrm{~s}^{-1}}\right)^{2 / 7}\left(\frac{n_{0}}{\mathrm{~cm}^{-3}}\right)^{-3 / 7}\left(\frac{\mu}{1.3}\right)^{-3 / 7} \\
& \left(\frac{E_{\mathrm{SN}}}{10^{51} \mathrm{erg}}\right)^{1 / 7} \mathrm{pc}
\end{aligned}
$$

and

$$
\begin{aligned}
u_{\mathrm{s}}\left(t_{\mathrm{f}}\right)=\frac{2}{5} \frac{R\left(t_{\mathrm{f}}\right)}{t_{\mathrm{f}}}= & 3.04\left(\frac{c}{1 \mathrm{~km} \mathrm{~s}^{-1}}\right)^{-3 / 7}\left(\frac{n_{0}}{\mathrm{~cm}^{-3}}\right)^{1 / 7} \\
& \left(\frac{\mu}{1.3}\right)^{1 / 7}\left(\frac{E_{\mathrm{SN}}}{10^{51} \mathrm{erg}}\right)^{2 / 7} \mathrm{~km} \mathrm{~s}^{-1} .
\end{aligned}
$$

\section{Two possible supernova explosions}

As we observe (Fig. 1), the OB stars are not uniformly distributed. They present different densities and holes. Here we chose two cases presenting circular arcs and to explain these as star groups formed by a triggered effect, by the supernova explosions. These approximately circular forms suggest that pre-stellar gas was uniformly swept up by a central source of pressure. We chose two circles with different size.

The small circle has a center at $\mathrm{RA}_{2000}=1^{\mathrm{h}} 16^{\mathrm{m}}$ and $\operatorname{Dec}_{2000}=-73^{\circ} 20^{\prime}$ and radius 32.4 pc. On the right part of this circle we find the end of the nebula N84 and on the left part the beginning of the $\mathrm{N} 85$.

The large circle has a center at $\mathrm{RA}_{2000}=1^{\mathrm{h}} 16^{\mathrm{m}}$ and $\mathrm{Dec}_{2000}=-73^{\circ} 11^{\prime}$ and radius $58.9 \mathrm{pc}$. At its center we find the association DEM156 and at its circumference the association DEM155, the nebula N86 and the associations DEM158 and DEM159.

Both circles have a radius less than $100 \mathrm{pc}$, so we can consider isolated supernova explosions. We suppose now that the radii of circles now are not so different from the radius at the fragmentation time. The small circle has a radius $R_{1}\left(t_{\mathrm{f}}\right)$ and the large circle a radius $R_{2}\left(t_{\mathrm{f}}\right)$. We also suppose that the average molecule in a cloud is $\mu=1.3$, the total energy of a supernova is $E_{\mathrm{SN}}=10^{51} \mathrm{erg}$ and the velocity dispersion in the shell is $c=1 \mathrm{~km} \mathrm{~s}^{-1}$. From Eq. (22) we have

$R\left(t_{\mathrm{f}}\right)=410.6\left(\frac{n_{0}}{\mathrm{~cm}^{-3}}\right)^{-3 / 7} \mathrm{pc}$ 
which gives

$n_{0}=\left(\frac{R\left(t_{\mathrm{f}}\right)}{410.6 \mathrm{pc}}\right)^{-7 / 3} \mathrm{~cm}^{-3}$.

Equation (25) gives for the small circle a numerical density before the explosion of $n_{01}=374 \mathrm{~cm}^{-3}$ and for the large circle $n_{02}=93 \mathrm{~cm}^{-3}$. From our star counts and the derived densities corresponding to the isopleths we have found that in the small circle the number density corresponding to the brightest stars with age about $5 \times 10^{6} \mathrm{yr}$ is 14 times the background value whereas in the large circle it is 3.2 times. The ratio of the two is about 5 with $30 \%$ error. The ratio in the above theoretical densities is 4 . This is a very good result considering the errors. The triggering star formation for the two possible supernova explosions has been verified by using the image of stellar densities. Isodensity contour plots present older B populations at the centers of our study regions (Fig. 2). These populations remain until the slice corresponding to main sequence $B$ stars with ages between $1.2 \times 10^{7}$ and $3 \times 10^{7} \mathrm{yr}$. That means that the two possible supernova explosions occurred about $3 \times 10^{7} \mathrm{yr}$ ago.

The diagrams of Fig. 2 are actually isopleths showing all main sequence stars at four levels of magnitude. We indicate at what magnitude level (marked in terms of age, Table 1) the OB stars disappear, as expected. In this case we determine the number of stars per unit pixel by using the values of background $b$ and standard deviation $\sigma$. The values used to determine these isopleths are: $b=0.2$ and $\sigma=0.29$ stars per pixel for the upper left, $b=0.59$ and $\sigma=0.33$ stars per pixel for the upper right, $b=0.71$ and $\sigma=0.66$ stars per pixel for the lower left and $b=1.1$ and $\sigma=0.68$ stars per pixel for the lower right. The isopleth density is given by $b+3 \sigma$. In the lower right case of Fig. 2 there are no isopleth density contours because the contrast is not high enough.

\section{Conclusions}

In this paper we attempt to show by a systematic observational method that it is possible to generate the birth of massive OB stars by triggered effects like supernova explosions. We have chosen the region N83-84-85 of the inner wing of SMC and we detected all the non-saturated OB stars in an objective prism plate of this region. We extracted and classified the stars automatically, using a method developed previously by us. The spatial distribution of the $\mathrm{OB}$ stars was found to be non-uniform with holes and high density parts that could be explained as star formation regions caused by supernovae. Two possible cases have been studied. The theoretical approach was the same as Ehlerová et al. (1997) but for isolated explosions.
From the observations we estimated the radius where the new population is born assuming that the supernova explosions occurred at their centers and from the theoretical equations we extracted the numerical densities of the ISM before the explosions. A study of isopleths of the stellar population of this region, from direct plates at various magnitude slices, provides an estimation of the time when the possible explosions took place. Finally the actual catalogue of the detected OB stars is given.

Acknowledgements. The authors are grateful to ROE for the loan of the observational material. The authors also thank F. Maragoudaki and E. Livanou for the contour programs. M.K. would like to thank the ELKE of the EKPAN (University of Athens) for financial support.

\section{References}

Azzopardi, M., \& Vigneau, J. 1982, A\&AS, 50, 291

Bica, E. L. D., \& Schmitt, H. R. 1995, ApJS, 101, 41

Bratsolis, E., Bellas-Velidis, I., Kontizas, E., et al. 1998, A\&AS, 133, 293

Bratsolis, E., Bellas-Velidis, I., Dapergolas, A., Kontizas, E., \& Kontizas, M. 2000, A\&AS, 142, 339

Bratsolis, E. 2002, in Automated Data Analysis in Astronomy, Narosa, ed. R. Gupta, H. P. Singh, \& C. A. L. Bailer-Jones, 99

Caplan, J., Taisheng, Ye, Deharveng, L., Turtle, A. J., \& Kennicutt, R. C. 1996, A\&A, 307, 403

Dapergolas, A., Kontizas, E., Kontizas, M., et al. 1991, A\&AS, 87, 97

Dyson, J. E., \& Williams, D. A. 1997, The Physics of Interstellar Medium, Second Edition (Bristol, Philadelphia: Institute of Physics Publishing)

Efremov, Y. N., \& Elmegreen, B. G. 1988, MNRAS, 299, 643

Elmegreen, B. G. 1994, ApJ, 427, 384

Ehlerová, S., Palous, J., Theis, Ch., \& Hensler, G. 1997, A\&A, 328, 121

Kahabka, P., Pietsch, W., Filipović, \& Heberl, F. 1999, A\&AS, 136, 81

Kontizas, E., Morgan, D. H., Kontizas, M., \& Dapergolas, A. 1988, A\&A, 201, 208

Lortet, M.-C., \& Testor, G. 1988, A\&A, 194, 11

Testor, G., \& Lortet, M.-C. 1987, A\&A, 178, 25

Maragoudaki, F., Kontizas, M., Kontizas, E., Dapergolas, A., \& Morgan, D. H. 1998, A\&A, 338, L29

Stanimirović, S., Staveley-Smith, L., Dickey, J. M., Sault, R. J., \& Snowden, S. L. 1999, MNRAS, 302, 417

Staveley-Smith, L., Sault, R. J., Hatzidimitriou, D., Kesteven, M. J., \& McConnel, D. 1997, MNRAS, 289, 225

Vishniac, E. T. 1994, ApJ, 428, 186

Westerlund, B. E. 1961, Uppsala Astron. Obs. Ann., 5, 2

Westerlund, B. E. 1997, The Megellanic Clouds, ed. A. King, D. Lin, S. Maran, J. Pringle, \& M. Ward (Cambridge University Press) 


\section{Online Material}


E. Bratsolis et al.: Triggered star formation in the inner wing of SMC, Online Material p 2

Table 3. Detected (non-saturated) OB stars in our region.

\begin{tabular}{|c|c|c|c|c|c|c|c|c|}
\hline Number & $\begin{array}{l}\mathrm{RA}(2000) \\
\mathrm{h} \mathrm{m} \mathrm{s}\end{array}$ & $\begin{array}{c}\operatorname{Dec}(2000) \\
\circ, \quad,\end{array}$ & Number & $\begin{array}{l}\mathrm{RA}(2000) \\
\mathrm{h} \mathrm{m} \mathrm{s}\end{array}$ & $\begin{array}{c}\operatorname{Dec}(2000) \\
\circ, \prime\end{array}$ & Number & $\begin{array}{l}\mathrm{RA}(2000) \\
\mathrm{h} \mathrm{m} \mathrm{s}\end{array}$ & $\begin{array}{c}\operatorname{Dec}(2000) \\
\circ, ",\end{array}$ \\
\hline 1 & 011858 & -733728 & 42 & 011735 & -733200 & 83 & 011745 & -732847 \\
\hline 2 & 011860 & -733708 & 43 & 011860 & -733126 & 84 & 011353 & -733004 \\
\hline 3 & 012047 & -733619 & 44 & 012019 & -733044 & 85 & 011752 & -732842 \\
\hline 4 & 011753 & -733724 & 45 & 012033 & -733035 & 86 & 011429 & -732951 \\
\hline 5 & 011634 & -733746 & 46 & 011805 & -733129 & 87 & 011614 & -732908 \\
\hline 6 & 011412 & -733823 & 47 & 011511 & -733229 & 88 & 011759 & -732823 \\
\hline 7 & 011839 & -733642 & 48 & 011815 & -733122 & 89 & 011916 & -732744 \\
\hline 8 & 012004 & -733604 & 49 & 012021 & -733018 & 90 & 011917 & -732743 \\
\hline 9 & 011417 & -733803 & 50 & 011901 & -733051 & 91 & 011931 & -732734 \\
\hline 10 & 011541 & -733718 & 51 & 011936 & -733035 & 92 & 011719 & -732824 \\
\hline 11 & 011238 & -733809 & 52 & 011608 & -733148 & 93 & 011840 & -732746 \\
\hline 12 & 011426 & -733735 & 53 & 011930 & -733028 & 94 & 011723 & -732808 \\
\hline 13 & 011755 & -733619 & 54 & 011511 & -733158 & 95 & 011904 & -732710 \\
\hline 14 & 011646 & -733636 & 55 & 011458 & -733159 & 96 & 011636 & -732805 \\
\hline 15 & 012045 & -733459 & 56 & 011760 & -733050 & 97 & 011809 & -732730 \\
\hline 16 & 012037 & -733459 & 57 & 011938 & -733008 & 98 & 011430 & -732832 \\
\hline 17 & 012036 & -733458 & 58 & 011938 & -733004 & 99 & 011455 & -732823 \\
\hline 18 & 012012 & -733503 & 59 & 011538 & -733131 & 100 & 011901 & -732653 \\
\hline 19 & 011604 & -733632 & 60 & 011342 & -733201 & 101 & 011503 & -732819 \\
\hline 20 & 011625 & -733609 & 61 & 011744 & -733037 & 102 & 011759 & $-7327 \quad 17$ \\
\hline 21 & 011931 & -733455 & 62 & 012003 & -732940 & 103 & 011958 & -732624 \\
\hline 22 & 011429 & -733638 & 63 & 011645 & -733056 & 104 & 012024 & -732605 \\
\hline 23 & 011646 & -733550 & 64 & 011905 & -732954 & 105 & 011547 & -732751 \\
\hline 24 & 011943 & -733431 & 65 & 011947 & -732937 & 106 & 011656 & -732726 \\
\hline 25 & 011944 & -733420 & 66 & 012038 & -732908 & 107 & 011858 & -732636 \\
\hline 26 & 011809 & -733425 & 67 & 011408 & -733125 & 108 & 011316 & -732833 \\
\hline 27 & 012005 & -733326 & 68 & 011427 & -733106 & 109 & 011746 & -732703 \\
\hline 28 & 011554 & -733447 & 69 & 011525 & -733047 & 110 & 011544 & -732746 \\
\hline 29 & 012019 & -733243 & 70 & 011846 & -732923 & 111 & 011502 & -732757 \\
\hline 30 & 011359 & -733455 & 71 & 011307 & -733114 & 112 & 011446 & -732756 \\
\hline 31 & 011238 & -733516 & 72 & 011454 & -733036 & 113 & 011540 & -732737 \\
\hline 32 & 011701 & -733332 & 73 & 011535 & -733020 & 114 & 011644 & -732710 \\
\hline 33 & 011945 & -733215 & 74 & 011757 & -732922 & 115 & 011424 & -732754 \\
\hline 34 & 011749 & -733260 & 75 & 011902 & -732853 & 116 & 011643 & -732706 \\
\hline 35 & 011832 & -733243 & 76 & 011705 & -732937 & 117 & 011615 & -732708 \\
\hline 36 & 011639 & -733319 & 77 & 011619 & -732948 & 118 & 011525 & -732724 \\
\hline 37 & 011653 & -733304 & 78 & 011722 & -732908 & 119 & 012038 & -732523 \\
\hline 38 & 011844 & -733204 & 79 & 012031 & -732745 & 120 & 011544 & -732701 \\
\hline 39 & 011731 & -733229 & 80 & 011633 & -732919 & 121 & 011710 & -732629 \\
\hline 40 & 011920 & -733139 & 81 & 011612 & -732923 & 122 & 011835 & -732556 \\
\hline 41 & 011453 & -733308 & 82 & 011248 & -733025 & 123 & 011459 & -732703 \\
\hline
\end{tabular}


E. Bratsolis et al.: Triggered star formation in the inner wing of SMC, Online Material p 3

Table 3. continued.

\begin{tabular}{|c|c|c|c|c|c|c|c|c|}
\hline Number & $\begin{array}{l}\mathrm{RA}(2000) \\
\mathrm{h} \mathrm{m} \mathrm{s}\end{array}$ & $\begin{array}{c}\operatorname{Dec}(2000) \\
\circ,{ }^{\prime}\end{array}$ & Number & $\begin{array}{l}\overline{R A(2000)} \\
h \text { m s }\end{array}$ & $\begin{array}{c}\operatorname{Dec}(2000) \\
\circ,{ }^{\prime}\end{array}$ & Number & $\begin{array}{l}\mathrm{RA}(2000) \\
\mathrm{h} \mathrm{m} \mathrm{s}\end{array}$ & $\begin{array}{c}\operatorname{Dec}(2000) \\
\circ, \quad,\end{array}$ \\
\hline 124 & 011411 & -732709 & 165 & 011859 & -732310 & 206 & 011943 & -732019 \\
\hline 125 & 011823 & -732536 & 166 & 011518 & -732424 & 207 & 011310 & -732232 \\
\hline 126 & 012005 & -732453 & 167 & 011713 & -732340 & 208 & 011456 & -732160 \\
\hline 127 & 011625 & -732619 & 168 & 011837 & -732301 & 209 & 011220 & -732242 \\
\hline 128 & 011352 & -732706 & 169 & 011819 & -732305 & 210 & 011222 & -732241 \\
\hline 129 & 011555 & -732624 & 170 & 012013 & -732212 & 211 & 011900 & -732026 \\
\hline 130 & 011711 & -732554 & 171 & 011536 & -732353 & 212 & 011848 & -732028 \\
\hline 131 & 011226 & -732719 & 172 & 012010 & -732206 & 213 & 011643 & $\begin{array}{llll}-73 & 21 & 12\end{array}$ \\
\hline 132 & 011417 & -732642 & 173 & 011943 & -732216 & 214 & 011922 & -732011 \\
\hline 133 & 011555 & -732559 & 174 & 011326 & -732424 & 215 & 011336 & -732210 \\
\hline 134 & 011460 & -732616 & 175 & 011536 & -732339 & 216 & 011907 & -732001 \\
\hline 135 & 011928 & -732437 & 176 & 011459 & -732349 & 217 & 012010 & -731932 \\
\hline 136 & 011600 & -732553 & 177 & 011515 & -732341 & 218 & 011328 & -732153 \\
\hline 137 & 011758 & -732502 & 178 & 011828 & -732231 & 219 & 011515 & -732121 \\
\hline 138 & 011950 & -732416 & 179 & 011632 & -732311 & 220 & 011550 & -732108 \\
\hline 139 & 011823 & -732446 & 180 & 011322 & -732404 & 221 & 011838 & -732006 \\
\hline 140 & 011323 & -732626 & 181 & 011932 & -732151 & 222 & 011748 & -732023 \\
\hline 141 & 011810 & -732448 & 182 & 011354 & -732350 & 223 & 011245 & -732156 \\
\hline 142 & 011507 & -732539 & 183 & 011635 & -732258 & 224 & 011629 & -732044 \\
\hline 143 & 011603 & -732520 & 184 & 011802 & -732219 & 225 & 011613 & -732049 \\
\hline 144 & 011805 & -732433 & 185 & 012024 & -732120 & 226 & 011609 & -732048 \\
\hline 145 & 011447 & -732539 & 186 & 011542 & -732252 & 227 & 011615 & -732043 \\
\hline 146 & 011606 & $\begin{array}{lll}-73 & 2512\end{array}$ & 187 & 011559 & -732244 & 228 & 011912 & -731934 \\
\hline 147 & 011331 & -732551 & 188 & 011609 & -732238 & 229 & 011339 & -732128 \\
\hline 148 & 011547 & -732505 & 189 & 011251 & -732335 & 230 & 011326 & -732131 \\
\hline 149 & 011553 & -732503 & 190 & 011653 & -732216 & 231 & 011315 & -732133 \\
\hline 150 & 011503 & -732515 & 191 & 011446 & -732257 & 232 & 011919 & -731925 \\
\hline 151 & 011246 & -732553 & 192 & 011448 & -732256 & 233 & 011853 & -731932 \\
\hline 152 & 011344 & -732536 & 193 & 011953 & -732103 & 234 & 012019 & -731857 \\
\hline 153 & 012004 & $\begin{array}{lll}-73 & 23 & 12\end{array}$ & 194 & 011352 & -732304 & 235 & 011532 & -732041 \\
\hline 154 & 011732 & -732413 & 195 & 011852 & -732112 & 236 & 011956 & -731858 \\
\hline 155 & 011730 & -732411 & 196 & 011827 & -732115 & 237 & 011940 & -731904 \\
\hline 156 & 011960 & -732307 & 197 & 011622 & -732159 & 238 & 011534 & -732035 \\
\hline 157 & 011759 & -732352 & 198 & 011820 & -732115 & 239 & 011833 & -731930 \\
\hline 158 & 011623 & -732427 & 199 & 011534 & -732204 & 240 & 011732 & -731953 \\
\hline 159 & 011329 & -732519 & 200 & 011620 & -732147 & 241 & 011751 & -731944 \\
\hline 160 & 011449 & -732455 & 201 & 011809 & -732106 & 242 & 011639 & -732010 \\
\hline 161 & 011339 & -732513 & 202 & 011655 & -732131 & 243 & 011520 & -732034 \\
\hline 162 & 011305 & -732517 & 203 & 011339 & -732233 & 244 & 011236 & -732121 \\
\hline 163 & 011508 & -732439 & 204 & 011650 & -732129 & 245 & 011922 & -731903 \\
\hline 164 & 011745 & -732341 & 205 & 011802 & -732059 & 246 & 011607 & -732010 \\
\hline
\end{tabular}


Table 3. continued.

\begin{tabular}{|c|c|c|c|c|c|c|c|c|}
\hline$\overline{\text { Number }}$ & $\begin{array}{l}\mathrm{RA}(2000) \\
\mathrm{h} \mathrm{m} \mathrm{s}\end{array}$ & $\begin{array}{c}\operatorname{Dec}(2000) \\
\circ,{ }^{\prime}\end{array}$ & Number & $\begin{array}{l}\text { RA(2000) } \\
\text { h m s }\end{array}$ & $\begin{array}{c}\operatorname{Dec}(2000) \\
\circ,{ }^{\prime}\end{array}$ & Number & $\begin{array}{l}\mathrm{RA}(2000) \\
\mathrm{h} \mathrm{m} \mathrm{s}\end{array}$ & $\begin{array}{c}\operatorname{Dec}(2000) \\
\circ,{ }^{\prime}\end{array}$ \\
\hline 247 & 011824 & -731917 & 288 & 011947 & -731706 & 329 & 011426 & -731710 \\
\hline 248 & 011742 & -731932 & 289 & 012027 & -731647 & 330 & 011243 & -731738 \\
\hline 249 & 011625 & -731957 & 290 & 011942 & -731706 & 331 & 011958 & -731455 \\
\hline 250 & 011601 & -732004 & 291 & 011641 & -731803 & 332 & 011803 & -731541 \\
\hline 251 & 011629 & -731954 & 292 & 011932 & -731657 & 333 & 011729 & -731553 \\
\hline 252 & 011755 & $\begin{array}{llll}-73 & 1922\end{array}$ & 293 & 011637 & -731757 & 334 & 011435 & -731646 \\
\hline 253 & 011235 & -732057 & 294 & 011606 & -731807 & 335 & 011828 & $\begin{array}{llll}-73 & 15 & 24\end{array}$ \\
\hline 254 & 011802 & $\begin{array}{llll}-73 & 19 & 10\end{array}$ & 295 & 011713 & $-73 \quad 1740$ & 336 & 011831 & -731515 \\
\hline 255 & 011759 & -731908 & 296 & 011441 & -731830 & 337 & 011815 & -731516 \\
\hline 256 & 011605 & -731948 & 297 & 011915 & -731651 & 338 & 011937 & -731443 \\
\hline 257 & 011558 & -731950 & 298 & 011636 & -731751 & 339 & 011231 & -73 1707 \\
\hline 258 & 011643 & -731934 & 299 & 011438 & -731826 & 340 & 011534 & -731611 \\
\hline 259 & 011455 & -732006 & 300 & 011903 & -731650 & 341 & 012021 & -731419 \\
\hline 260 & 011635 & -731932 & 301 & 011628 & -731745 & 342 & 011624 & -731547 \\
\hline 261 & 011433 & -732003 & 302 & 011934 & -731631 & 343 & 011727 & -731524 \\
\hline 262 & 012012 & -731754 & 303 & 011445 & -731815 & 344 & 011734 & -731519 \\
\hline 263 & 011308 & -732023 & 304 & 011747 & -731711 & 345 & 011751 & -731508 \\
\hline 264 & 011309 & -732023 & 305 & 011801 & -731701 & 346 & 011440 & -731612 \\
\hline 265 & 011547 & -731934 & 306 & 011702 & -731721 & 347 & 012005 & -731411 \\
\hline 266 & 011640 & -731916 & 307 & 011541 & -731749 & 348 & 011955 & -731404 \\
\hline 267 & 011542 & -731934 & 308 & 011604 & -731740 & 349 & 011529 & -731537 \\
\hline 268 & 011447 & -731945 & 309 & 011624 & -731728 & 350 & 011643 & -731507 \\
\hline 269 & 011535 & -731926 & 310 & 011743 & -731659 & 351 & 011417 & -731549 \\
\hline 270 & 011540 & -731925 & 311 & 011219 & -731840 & 352 & 011823 & -731424 \\
\hline 271 & 012017 & -731734 & 312 & 011543 & -731740 & 353 & 011625 & -731506 \\
\hline 272 & 011923 & -731756 & 313 & 011630 & -731722 & 354 & 012018 & -731333 \\
\hline 273 & 011602 & -731910 & 314 & 011709 & -731708 & 355 & 011932 & -731352 \\
\hline 274 & 011553 & -731910 & 315 & 011628 & -731716 & 356 & 011943 & -731345 \\
\hline 275 & 011555 & -731908 & 316 & 011715 & -731659 & 357 & 011629 & -731455 \\
\hline 276 & 011837 & -731809 & 317 & 011910 & -731614 & 358 & 012016 & -731326 \\
\hline 277 & 011755 & -731815 & 318 & 011421 & -731751 & 359 & 011352 & -731544 \\
\hline 278 & 011909 & -731745 & 319 & 011937 & -731550 & 360 & 011812 & -731416 \\
\hline 279 & 011533 & -731903 & 320 & 011533 & -731719 & 361 & 011816 & -731412 \\
\hline 280 & 011915 & -731741 & 321 & 011516 & -731723 & 362 & 011520 & -731511 \\
\hline 281 & 011718 & -731824 & 322 & 011328 & -731755 & 363 & 011654 & -731439 \\
\hline 282 & 011908 & -731741 & 323 & 011644 & -731649 & 364 & 011458 & $-73 \quad 1512$ \\
\hline 283 & 011258 & -731944 & 324 & 011226 & -731808 & 365 & 011941 & -731326 \\
\hline 284 & 011617 & -731841 & 325 & 011312 & -731751 & 366 & 011532 & -731457 \\
\hline 285 & 011236 & -731947 & 326 & 011658 & -731634 & 367 & 011526 & -731450 \\
\hline 286 & 011645 & -731825 & 327 & 011246 & -731746 & 368 & 011557 & -731439 \\
\hline 287 & 012010 & -731659 & 328 & 012010 & -731511 & 369 & 012005 & -731303 \\
\hline
\end{tabular}


E. Bratsolis et al.: Triggered star formation in the inner wing of SMC, Online Material p 5

Table 3. continued.

\begin{tabular}{|c|c|c|c|c|c|c|c|c|}
\hline Number & $\begin{array}{l}\mathrm{RA}(2000) \\
\mathrm{h} \mathrm{m} \mathrm{s}\end{array}$ & $\begin{array}{c}\operatorname{Dec}(2000) \\
\circ,{ }^{\prime}\end{array}$ & Number & $\begin{array}{l}\overline{R A(2000)} \\
h \text { m s }\end{array}$ & $\begin{array}{c}\operatorname{Dec}(2000) \\
\circ, \quad,\end{array}$ & Number & $\begin{array}{l}\mathrm{RA}(2000) \\
\mathrm{h} \mathrm{m} \mathrm{s}\end{array}$ & $\begin{array}{c}\operatorname{Dec}(2000) \\
\circ, \quad,\end{array}$ \\
\hline 370 & $01 \quad 1818$ & -731344 & 411 & 011438 & -731304 & 452 & 011720 & -731045 \\
\hline 371 & 011706 & -731409 & 412 & 011842 & -731139 & 453 & 011859 & -731006 \\
\hline 372 & 011252 & -731520 & 413 & 012006 & -731059 & 454 & 011659 & -731051 \\
\hline 373 & 011945 & -731258 & 414 & 011701 & -731210 & 455 & 011711 & -731047 \\
\hline 374 & 011308 & -731515 & 415 & 011454 & -731251 & 456 & 011933 & -730950 \\
\hline 375 & 011744 & -731339 & 416 & 011546 & -731235 & 457 & 011757 & -731023 \\
\hline 376 & 011752 & -731328 & 417 & 011600 & -731228 & 458 & 011750 & -731019 \\
\hline 377 & 012023 & -731220 & 418 & 011730 & -731156 & 459 & 011724 & $\begin{array}{llll}-73 & 1027\end{array}$ \\
\hline 378 & 011732 & -731326 & 419 & 011904 & -731116 & 460 & 011553 & -731049 \\
\hline 379 & 011647 & -731340 & 420 & 011340 & -731304 & 461 & 011743 & -731003 \\
\hline 380 & 011606 & -731354 & 421 & 011857 & -731109 & 462 & 011809 & -730953 \\
\hline 381 & 011727 & -731322 & 422 & 011541 & -731220 & 463 & 011850 & -730935 \\
\hline 382 & 011722 & -731321 & 423 & 011828 & -731120 & 464 & 011236 & -731132 \\
\hline 383 & 011854 & -731242 & 424 & 011949 & -731047 & 465 & 011656 & -731012 \\
\hline 384 & 011321 & -731433 & 425 & 011729 & -731142 & 466 & 011540 & -73 1037 \\
\hline 385 & 011539 & -731350 & 426 & 011744 & -731135 & 467 & 011557 & -731031 \\
\hline 386 & 011918 & -731223 & 427 & 011854 & -731108 & 468 & 011945 & -730902 \\
\hline 387 & 011951 & -731209 & 428 & 011458 & -731232 & 469 & 011410 & -731101 \\
\hline 388 & 011730 & -731301 & 429 & 011325 & -731259 & 470 & 011812 & -730937 \\
\hline 389 & 011756 & -731251 & 430 & 011930 & -731043 & 471 & 011818 & -730928 \\
\hline 390 & 011943 & -731204 & 431 & 011755 & -731118 & 472 & 011355 & -731053 \\
\hline 391 & 011649 & -731309 & 432 & 011912 & -731047 & 473 & 011427 & -731039 \\
\hline 392 & 011903 & $\begin{array}{lll}-73 & 12 & 14\end{array}$ & 433 & 011658 & -731137 & 474 & 011942 & -730846 \\
\hline 393 & 011253 & -731418 & 434 & 011937 & -731035 & 475 & 011842 & -730909 \\
\hline 394 & 011935 & -731156 & 435 & 011848 & -731054 & 476 & 011405 & -731042 \\
\hline 395 & 011435 & -731344 & 436 & 011335 & -731234 & 477 & 011702 & -730942 \\
\hline 396 & 011822 & -731223 & 437 & 011327 & -731235 & 478 & 011650 & -730939 \\
\hline 397 & 011534 & -731322 & 438 & 011537 & -731155 & 479 & 011619 & -730949 \\
\hline 398 & 011920 & -731159 & 439 & 011323 & -731228 & 480 & 011424 & -731023 \\
\hline 399 & 011936 & -731150 & 440 & 011352 & -731220 & 481 & 011508 & -731010 \\
\hline 400 & 011651 & -731251 & 441 & 011257 & -731234 & 482 & 011816 & -730904 \\
\hline 401 & 011440 & -731333 & 442 & 011615 & -731134 & 483 & 011528 & -731001 \\
\hline 402 & 011559 & -731305 & 443 & 011531 & -731145 & 484 & 011543 & -730955 \\
\hline 403 & 011431 & -731331 & 444 & 011332 & -731220 & 485 & 011541 & -730953 \\
\hline 404 & 011901 & -731156 & 445 & 011619 & -731125 & 486 & 011519 & -730958 \\
\hline 405 & 011416 & -731329 & 446 & 011704 & -731110 & 487 & 011725 & -730915 \\
\hline 406 & 011711 & -731222 & 447 & 011731 & -731051 & 488 & 011934 & -730822 \\
\hline 407 & 011740 & -731211 & 448 & 012018 & -730943 & 489 & 011442 & -731007 \\
\hline 408 & 011814 & -731157 & 449 & 011209 & -731224 & 490 & 011648 & -730924 \\
\hline 409 & 012011 & -731104 & 450 & 011416 & -731149 & 491 & 011907 & -730826 \\
\hline 410 & 011609 & -731236 & 451 & 011907 & -731006 & 492 & 011746 & -730856 \\
\hline
\end{tabular}


Table 3. continued.

\begin{tabular}{|c|c|c|c|c|c|c|c|c|}
\hline Number & $\begin{array}{l}\text { RA(2000) } \\
\text { h m s }\end{array}$ & $\begin{array}{c}\operatorname{Dec}(2000) \\
0, \quad,\end{array}$ & Number & $\begin{array}{l}\text { RA(2000) } \\
\text { h m s }\end{array}$ & $\begin{array}{c}\operatorname{Dec}(2000) \\
0, \quad,\end{array}$ & Number & $\begin{array}{l}\text { RA(2000) } \\
\text { h m s }\end{array}$ & $\begin{array}{c}\operatorname{Dec}(2000) \\
\circ \quad, \quad,\end{array}$ \\
\hline 493 & 011624 & -730925 & 534 & 011401 & -730802 & 575 & 011346 & -730501 \\
\hline 494 & 011420 & -731003 & 535 & 011432 & -730750 & 576 & 012005 & -730245 \\
\hline 495 & 011517 & -730944 & 536 & 011755 & -730641 & 577 & 011913 & -730303 \\
\hline 496 & 011537 & -730937 & 537 & 011748 & -730638 & 578 & 011709 & -730348 \\
\hline 497 & 011644 & -730908 & 538 & 012008 & -730541 & 579 & 011911 & -730255 \\
\hline 498 & 011530 & -730933 & 539 & 011936 & -730549 & 580 & 011910 & -730254 \\
\hline 499 & 011827 & -730828 & 540 & 011942 & -730546 & 581 & 011307 & -730455 \\
\hline 500 & 011931 & -730758 & 541 & 011933 & -730545 & 582 & 011744 & -730320 \\
\hline 501 & 011319 & -731005 & 542 & 011515 & -730719 & 583 & 011327 & -730438 \\
\hline 502 & 011348 & -730954 & 543 & 011410 & -730733 & 584 & 011459 & -730410 \\
\hline 503 & 011915 & -730759 & 544 & 011322 & -730737 & 585 & 011658 & -730326 \\
\hline 504 & 011654 & -730849 & 545 & 012009 & -730513 & 586 & 011641 & -730331 \\
\hline 505 & 011602 & -730859 & 546 & 011656 & -730628 & 587 & 011351 & -730419 \\
\hline 506 & 011550 & -730902 & 547 & 011942 & -730519 & 588 & 011207 & -730439 \\
\hline 507 & 011409 & -730933 & 548 & 011719 & -730605 & 589 & 011724 & -730257 \\
\hline 508 & 011638 & -730843 & 549 & 011920 & -730517 & 590 & 011329 & -730406 \\
\hline 509 & 011556 & -730855 & 550 & 011415 & -730659 & 591 & 011604 & -730239 \\
\hline 510 & 011648 & -730837 & 551 & 011748 & -730545 & 592 & 011856 & -730130 \\
\hline 511 & 011416 & -730923 & 552 & 011550 & -730621 & 593 & 011429 & -730302 \\
\hline 512 & 011622 & -730843 & 553 & 011443 & -730639 & 594 & 011558 & -730224 \\
\hline 513 & 011801 & -730805 & 554 & 011956 & -730444 & 595 & 011939 & -730059 \\
\hline 514 & 011716 & -730813 & 555 & 011813 & -730520 & 596 & 011728 & -730141 \\
\hline 515 & 011521 & -730851 & 556 & 011223 & -730711 & 597 & 011434 & -730232 \\
\hline 516 & 011811 & -730748 & 557 & 011612 & -730547 & 598 & 011942 & -730024 \\
\hline 517 & 011337 & -730910 & 558 & 011756 & -730510 & 599 & 011237 & -730240 \\
\hline 518 & 012007 & -730652 & 559 & 011338 & -730634 & 600 & 011608 & -730111 \\
\hline 519 & 011435 & -730849 & 560 & 011934 & -730427 & 601 & 011438 & -730134 \\
\hline 520 & 011360 & -730858 & 561 & 011820 & -730447 & 602 & 011205 & -730214 \\
\hline 521 & 011626 & -730812 & 562 & 011560 & -730519 & 603 & 011420 & -730132 \\
\hline 522 & 011633 & -730805 & 563 & 011804 & -730435 & 604 & 011221 & -730139 \\
\hline 523 & 011917 & -730648 & 564 & 011642 & -730501 & 605 & 011615 & -730022 \\
\hline 524 & 011753 & -730717 & 565 & 011712 & -730444 & 606 & 011960 & -735855 \\
\hline 525 & 011701 & -730735 & 566 & 011906 & -730349 & 607 & 011752 & -735944 \\
\hline 526 & 011746 & -730713 & 567 & 011248 & -730548 & 608 & 011320 & -730108 \\
\hline 527 & 011724 & -730719 & 568 & 011801 & -730402 & 609 & 011329 & -730106 \\
\hline 528 & 011559 & -730747 & 569 & 011601 & -730441 & 610 & 011541 & -730026 \\
\hline 529 & 011451 & -730808 & 570 & 011619 & -730428 & & & \\
\hline 530 & 011313 & -730826 & 571 & 011613 & -730428 & & & \\
\hline 531 & 011614 & -730729 & 572 & 011515 & -730447 & & & \\
\hline 532 & 011213 & -730834 & 573 & 011538 & -730434 & & & \\
\hline 533 & 011525 & -730737 & 574 & 011845 & -730325 & & & \\
\hline
\end{tabular}

